\section{Evaluación de la calidad de vida de pacientes bipolares chilenos: propiedades psicométricas y utilidad diagnóstica de la versión chilena del cuestionario Quality of Life Bipolar Disorder (QoL.BD-CL)}

\author{
CAROLINA MORGADO ${ }^{1, a}$, TAMARA TAPIA ${ }^{1, a}$, \\ FERNANDO IVANOVIC-ZUVIC ${ }^{1,2}$, ANDRÉS ANTIVILO ${ }^{1, a, b}$
}

\section{Assessment of a version adapted and translated into Spanish of the Quality of Life Bipolar Disorder Questionnaire}

Background: The Quality of life Bipolar Disorder (QoL.BD) Questionnaire specifically measures quality of life in patients with bipolar disorder. Aim: To adapt a version translated into Spanish of the questionnaire and assess its validity in Chilean patients. Material and Methods: The QoL. BD was adapted to the Chilean population through the back-translation method and then administered to 32 adult patients with a bipolar disorder and 31 subjects without the disease, both groups with similar socioeconomic status. To confirm the diagnosis, the International Neuropsychiatric Interview (MINI), Young (YMRS) and Hamilton (HAM-D) scales were applied. Quality of life was assessed using the SF-36v.2 survey. We determined internal consistency, reliability, convergent validity, the cut-off point, and the sensibility and specificity of the scale. Results: The Chilean version of the Questionnaire [QoL. BD-CL] had a high reliability $(\alpha=0.95)$ and a high validity in reference to external criteria (correlation coefficients with SF-36 ranging from 0.453 and $0.819 ; p<0.01$ ). A cut-off point of 170 , with sensitivity of $87.9 \%$ and specificity of $80 \%$ was determined. Conclusions: QoL. $B D-C L$ has adequate psychometric properties, as well as an adequate sensitivity and specificity to distinguish between negative and positive perceptions of life quality in Chilean patients with bipolar disorders.

(Rev Med Chile 2015; 143: 213-222)

Key words: Bipolar disorder; Questionnaires.

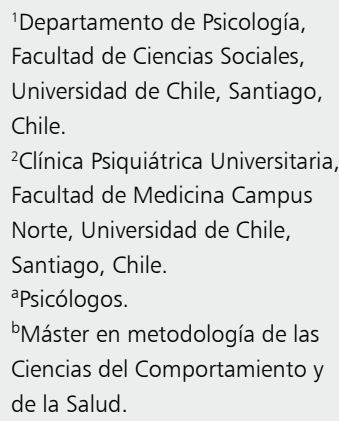

Recibido el 12 de abril de 2014, aceptado el 22 de noviembre de 2014.

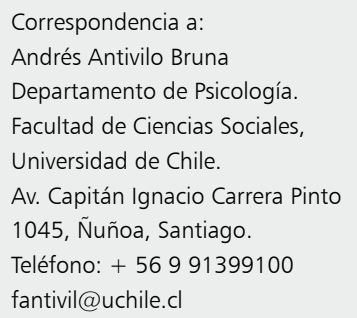

\footnotetext{
T os trastornos bipolares (TB) son definidos como patologías de la regulación del estado $\checkmark$ de ánimo, con un curso recurrente y diversas formas de presentación ${ }^{1}$. Son la sexta causa mundial de carga social y sufrimiento, se ubican en el quinto lugar de las enfermedades que causan discapacidad en jóvenes ${ }^{2}$ y afectan entre $0,5 \%$ a $5 \%$ de la población ${ }^{1,3}$.
}

En relación a los efectos psicosociales, los TB provocan un mayor deterioro en esta área que la depresión unipolar, afectándose en mayor medida los ámbitos laboral, social y familiar ${ }^{4,5}$. La tasa de mortalidad fluctúa entre 15 a 20\%, llegando a ser la enfermedad con mayor riesgo suicida junto con la depresión monopolar ${ }^{1}$. Considerando lo anterior, en Chile a partir del año 2013 el TB es 
ingresado al Plan de Acceso Universal de Garantías Explícitas [AUGE], plan que declara como uno de los objetivos principales de las intervenciones psicosociales la mejora de la calidad de vida de las personas ${ }^{6}$. Al respecto, la Organización Mundial de la Salud (OMS) define calidad de vida como la "percepción que tiene un individuo de su posición en la vida dentro del contexto cultural y valórico en que vive y en relación a sus objetivos, expectativas, estándares y preocupaciones" ${ }^{\prime}$. Este concepto también puede aplicarse en el ámbito de la salud, midiéndose cómo una enfermedad crónica puede afectar la vida del paciente, lo que es entendido como calidad de vida relacionada con la salud (CVRS) $)^{8,9,10,11}$.

De acuerdo a Michalak et al $^{12}$, el instrumento más utilizado para evaluar la CVRS es la encuesta de salud SF-36 y sus distintas versiones, las cuales abordan el concepto de manera general. No obstante, era necesario contar con un instrumento específico para evaluar CVRS en pacientes bipolares, lo que originó el desarrollo del Quality of life in Bipolar Disorder (QoL. BD) Questionnaire en Canadá. Dicho cuestionario se validó con 225 pacientes adultos, encontrándose correlaciones con el SF-36 que variaban entre 0,35 y 0,77; además se registraron adecuados valores de fiabilidad en sus dimensiones ( $\alpha$ entre 0,79 y 0,95$)^{13}$.

Mediante este instrumento es posible establecer cuál área de la vida de los pacientes bipolares requiere mayor atención, lo que ayuda a los profesionales de la salud mental a definir los objetivos para el tratamiento y apoya a las investigaciones en comprender los efectos de los diferentes tratamientos aplicados para este trastorno ${ }^{13}$.

De este modo, el objetivo del presente estudio es tanto adaptar al español como determinar las propiedades psicométricas (evidencias de validez y fiabilidad) y la utilidad diagnóstica del cuestionario QoL. BD, para medir la calidad de vida en pacientes bipolares tipo I y II, chilenos, atendidos en centros de salud públicos y privados, de ambos sexos, entre 18 y 65 años.

\section{Material y Método}

\section{Instrumento}

El QoL. BD es un cuestionario auto-administrado que consta de 12 dimensiones obligatorias (Estado físico, Sueño, Ánimo, Cognición, Ocio,
Vida social, Espiritualidad, Finanzas, Vida doméstica, Autoestima, Independencia e Identidad) y dos opcionales (Trabajo y Educación), cada una de las cuales contiene cuatro ítems (56 ítems en total) (ver Anexo 1). Es una escala tipo Likert con cinco opciones graduadas de respuesta, donde se le pide al paciente que conteste en referencia a su experiencia durante los últimos siete días ${ }^{14}$. La interpretación del cuestionario está dada por el formato de respuesta y se establece para cada dimensión. Por ejemplo, una persona que obtiene un promedio de cinco puntos en la dimensión vida doméstica, indica que está muy de acuerdo con la satisfacción de esa dimensión de su calidad de vida ${ }^{14}$.

\section{Participantes}

Se realizó un muestreo no probabilístico por conveniencia y pareado. Se trabajó con dos grupos: (i) Grupo clínico: 32 pacientes bipolares ambulatorios reclutados en la Clínica Universitaria de la Universidad de Chile y en el Hospital del Salvador, ambos ubicados en Santiago de Chile. El diagnóstico de trastorno bipolar (tipo I y II) fue realizado previamente por un médico psiquiatra y confirmado, para efectos de esta investigación, mediante tres instrumentos de pesquisa: International Neuropsychiatric Interview (MINI), Escala de Young (YMRS) y Escala de Hamilton (HAM-D). Cabe señalar que se utilizaron similares criterios de inclusión que el estudio original, por lo que no todos los sujetos estudiados estaban en estado de eutimia, según los criterios tanto de la YMRS como de la escala HAM-D. De acuerdo a la información obtenida, el grupo quedó finalmente conformado por 17 pacientes TB tipo I y 15 pacientes TB tipo II. Además, en el momento de la entrevista 26 de estos pacientes presentaron remisión sintomática del polo maníaco y seis reportaron sintomatología maníaca en distinto grado de intensidad. Por otra parte, 15 pacientes no presentaron sintomatología depresiva y 17 reportaron síntomas depresivos de leve a grave; (ii) Grupo no clínico: 31 sujetos con las mismas características sociodemográficas que el grupo clínico y sin diagnóstico de trastorno bipolar.

\section{Evaluación y material utilizado}

A todos los participantes se les aplicaron los tres instrumentos de pesquisa psiquiátrica ya mencionados (MINI, YMRS y HAM-D), y para la 
evaluación de la calidad de vida se administraron el cuestionario QoL. BD en su versión en español (QoL. BD-CL, de ahora en adelante) y la encuesta SF-36v.2, validada previamente en Chile por Olivares-Tirado ${ }^{15}$.

\section{Procedimiento y análisis de datos}

En primer lugar se realizó el proceso de adaptación y traducción al español del cuestionario QoL. BD. En segundo lugar, se desarrolló la aplicación piloto del test y, una vez revisados los posibles problemas en la comprensión de la versión adaptada, se realizó la aplicación experimental. En ambas ocasiones se les administraron a todos los participantes el formato de consentimiento informado, y los instrumentos de pesquisa psiquiátrica y cuestionarios de calidad de vida ya descritos. Los datos sociodemográficos y la MINI se ingresaron al momento de la entrevista en el programa Epidata 3.1 para su posterior exportación al programa Statistical package for the social sciences [IBM SPSS 20]. La información de las escalas HAM-D, YMRS y de los dos cuestionarios de calidad de vida se ingresaron una vez concluida la entrevista en el programa SPSS 20. Para obtener evidencias de validez referida a criterio externo (validez concurrente) se calculó, entre las dimensiones del QoL. BD-CL y el SF-36v.2, el coeficiente de correlación producto-momento de Pearson con su correspondiente coeficiente de determinación. Asimismo, para obtener indicadores del nivel de confiabilidad del cuestionario se estableció el valor del coeficiente alpha de Cronbach. A continuación, tanto los puntajes del QoL. BD-CL como del SF-36 se sometieron a un análisis con curvas ROC (Receiver Operator Characteristic) para estimar los puntos de corte que entregan información sobre la sensibilidad y la especificidad, determinándose el área bajo la curva para establecer la utilidad diagnóstica de ambos cuestionarios. Luego, y con la finalidad de establecer si hay diferencias entre los grupos clínico y no clínico, se utilizó la prueba t de Student para comparar las variables cuantitativas (las puntuaciones en los cuestionarios y la edad) y la prueba $\chi^{2}$ de Pearson para las variables categóricas (sexo, estado civil y nivel educacional). Por último, se debe destacar que el estudio fue aprobado por el Comité de Ética del Servicio de Salud Metropolitano Oriente y el Comité de Ética de la Clínica Universitaria de la Universidad de Chile.

\section{Resultados}

\section{Adaptación y transculturización}

Se utilizó el diseño back translation, el cual consistió en traducir el cuestionario del inglés al español por dos psicólogos de modo independiente. Ambas traducciones fueron discutidas y consensuadas por el equipo de investigación y los traductores. Posteriormente, la versión resultante fue retraducida al inglés por otros dos traductores, llegando de la misma manera a una versión consensuada en inglés, la cual fue aprobada por los autores del instrumento. A partir del pilotaje de dicha versión, se decidió modificar el formato de las preguntas filtros de las dimensiones opcionales y se estimó que el tiempo promedio de aplicación es de $7 \mathrm{~min}$.

\section{Descripción de los grupos de estudio}

En la Tabla 1 se resumen las características sociodemográficas de los dos grupos estudiados. Se puede observar que no hay diferencias significativas en sexo, edad, estado civil y nivel educacional ( $\mathrm{p}$ $>0,05$ en todos los contrastes), lo que permite afirmar que ambos grupos son homogéneos. El grupo clínico percibió una peor calidad de vida en todas las dimensiones del QoL. BD-CL (Figura 1a) obteniendo un promedio de $145,3 \pm 33$ puntos, frente a un 196,5 $\pm 22,9$ puntos del grupo no clínico, diferencia estadísticamente significativa $(\mathrm{t}=-7,1 ; \mathrm{p}<0,001)$. Asimismo, los resultados obtenidos con el cuestionario SF-36v.2 (Figura 1b) dan cuenta de una diferencia significativa entre ambos grupos, tanto en el componente de salud mental (CSM) $(\mathrm{t}=-8,5 ; \mathrm{p}<0,001)$ como en el de salud físico (CSF) $(t=-6,7 ; p<0,001)$, donde el grupo de pacientes bipolares reportó una peor calidad de vida $(\mathrm{CSM}=45 \pm 17,2$ y $\mathrm{CSF}=64,9 \pm$ $19,8)$ que el grupo no clínico (CSM $=78,6 \pm 13,8$ y CSF $=89,5 \pm 6,1)$.

\section{Validez referida a criterio externo}

Se estimó a partir de la asociación observada entre las 14 dimensiones del QoL. BD-CL y las 8 dimensiones del SF-36v.2, además del CSF, CSM y la pregunta del estado de transición (Tabla 2). Los puntajes totales del QoL. BD-CL (considerando sus versiones de 48 y 56 ítems) se relacionaron de manera directa y significativa $(\mathrm{p}<0,01)$ con ambos componentes de salud del SF-36v.2. La correlación más alta se obtuvo entre el total del QoL. BD-CL (versión de 56 ítems) y el CSM $(r=0,819)$. 
Tabla 1. Características Sociodemográficas de la muestra

\begin{tabular}{|c|c|c|c|c|}
\hline \multirow[b]{2}{*}{ Parámetros } & \multicolumn{2}{|c|}{ Descriptivos según grupos } & \multicolumn{2}{|c|}{ Comparaciones por grupo } \\
\hline & Grupo clínico & Grupo no clínico & Estadístico & Sig. \\
\hline \multicolumn{5}{|l|}{ Sexo } \\
\hline$\%$ Mujeres & $68,8(22)$ & $71 \quad(22)$ & $\chi^{2}=0,037$ & 0,848 \\
\hline$\%$ Hombres & $31,3(10)$ & $29 \quad(9)$ & & \\
\hline Edad (años) & $\bar{x}=38 \pm 12,5$ & $\bar{x}=37 \pm 12,3$ & $t=0,144$ & 0,886 \\
\hline \multicolumn{5}{|l|}{ Estado civil } \\
\hline$\%$ Solteros & $62,5(20)$ & $64,5(20)$ & & \\
\hline$\%$ Casados & $21,9 \quad(7)$ & $25,8 \quad(8)$ & $\chi^{2}=0,134$ & $0, / 14$ \\
\hline \% Separados o divorciados & $15,6 \quad(5)$ & $9,7 \quad(3)$ & & \\
\hline \multicolumn{5}{|l|}{ Nivel educacional } \\
\hline$\%$ Media incompleta & $6,3 \quad(2)$ & $6,5 \quad(2)$ & & \\
\hline$\%$ Media completa & $6,3 \quad(2)$ & $6,5 \quad(2)$ & $\chi^{2}=0,002$ & 0,962 \\
\hline$\%$ Superior incompleta & $31,3(10)$ & $29 \quad(9)$ & & \\
\hline \% Superior completa & $56,3(18)$ & $58,1(18)$ & & \\
\hline
\end{tabular}
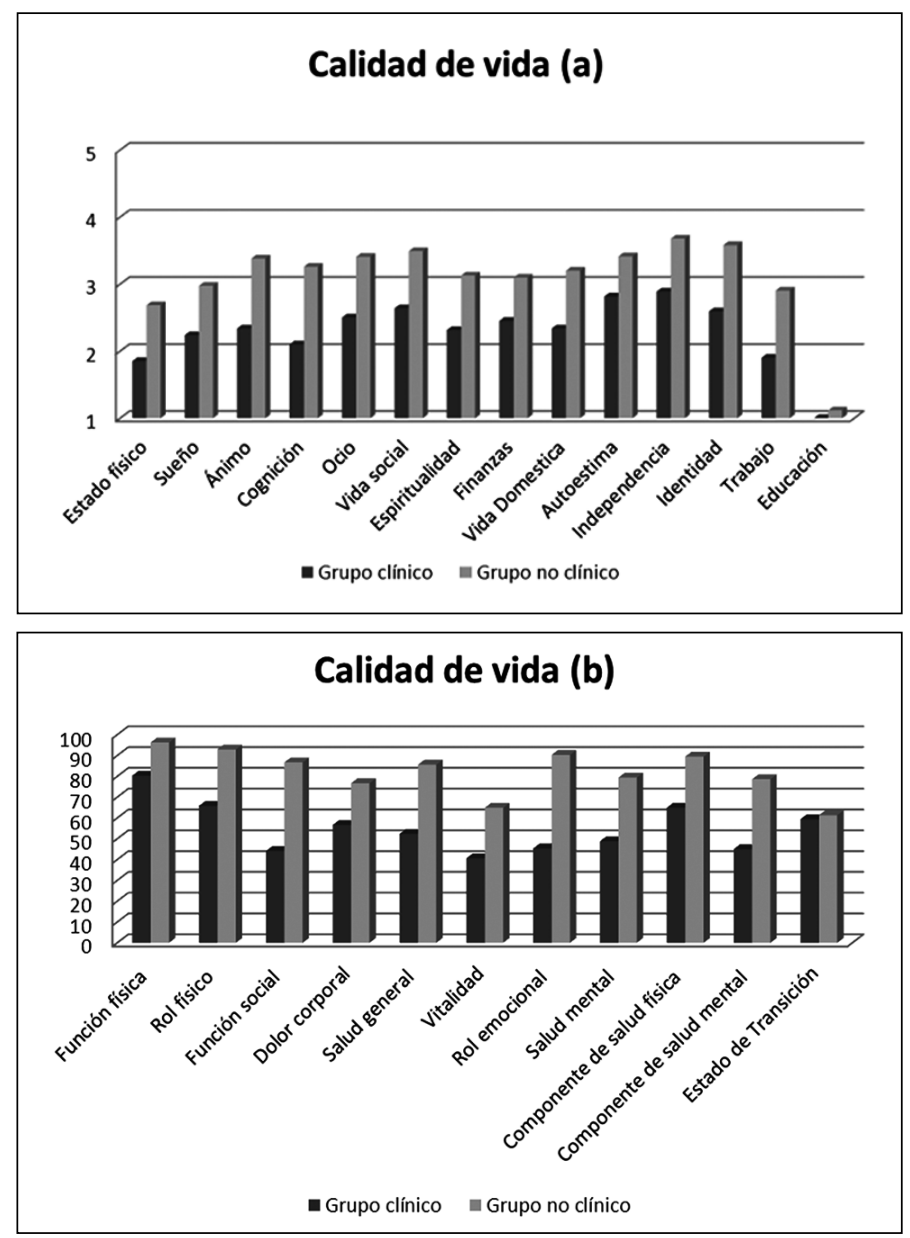

Figura 1. Comparación de puntajes entre QoL. $\mathrm{BD}-\mathrm{CL}$ y SF-36. (a) Media de las dimensiones del QoL. BD-CL en cada grupo. (b) Media de las dimensiones del SF-36 en cada grupo. 
Tabla 2. Correlaciones entre el QoL. BD-CL y el SF-36

\begin{tabular}{|c|c|c|c|c|c|c|c|c|c|c|c|c|}
\hline \multirow{17}{*}{ 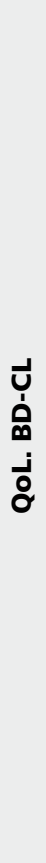 } & & CSF & CSM & $\mathbf{F F}$ & RF & DC & $\begin{array}{r}\mathbf{F} 6 \\
\text { SG }\end{array}$ & FS & VT & RE & SM & ET \\
\hline & EF & $0,698^{* *}$ & $0,768 * *$ & $0,642 * *$ & $0,526^{* *}$ & $0,418^{*}$ & $0,592 * *$ & $0,536^{* *}$ & $0,744^{* *}$ & $0,589 * *$ & $0,592^{* *}$ & $0,363^{*}$ \\
\hline & SU & $0,608^{* *}$ & $0,67^{* *}$ & $0,576^{* *}$ & $0,426^{*}$ & $0,569 * *$ & 0,441 * & $0,523 * *$ & $0,679 * *$ & $0,453^{* *}$ & $0,512^{* *}$ & - \\
\hline & AN & $0,465^{* *}$ & $0,661 * *$ & $0,433^{*}$ & - & - & $0,488^{* *}$ & 0,421 * & $0,575^{* *}$ & 0,371 * & $0,686 * *$ & $0,508^{* *}$ \\
\hline & COG & $0,639 * *$ & $0,683^{* *}$ & $0,537 * *$ & $0,458^{* *}$ & $0,408^{*}$ & $0,589 * *$ & $0,593^{* *}$ & $0,492 * *$ & $0,535^{*}$ & $0,641 * *$ & $0,385^{*}$ \\
\hline & $O C$ & $0,606^{* *}$ & $0,495^{* *}$ & $0,554^{* *}$ & - & $0,533^{* *}$ & $0,575^{* *}$ & $0,495^{* *}$ & - & - & $0,413^{*}$ & $0,45^{* *}$ \\
\hline & VS & - & - & - & - & - & - & $0,625^{* *}$ & - & - & - & - \\
\hline & ES & $0,572 * *$ & $0,745^{* *}$ & 0,441 * & $0,398^{*}$ & 0,391 * & $0,557^{* *}$ & $0,57^{* *}$ & $0,682^{* *}$ & $0,497^{* *}$ & $0,631 * *$ & $0,392^{*}$ \\
\hline & FI & - & - & - & - & - & $0,356^{*}$ & $0,439^{*}$ & - & - & - & - \\
\hline & VD & - & - & - & $0,365^{*}$ & - & - & $0,366^{*}$ & $0,381 *$ & - & - & - \\
\hline & $\mathbf{A U}$ & $0,498^{* *}$ & $0,676^{* *}$ & 0,449 * & - & - & $0,501 * *$ & $0,571^{* *}$ & $0,588^{* *}$ & - & $0,667^{* *}$ & $0,375^{*}$ \\
\hline & IN & $0,566^{* *}$ & $0,539 * *$ & $0,477^{* *}$ & - & $0,412^{*}$ & $0,567^{* *}$ & $0,469 * *$ & $0,459 * *$ & $0,370 *$ & $0,436^{*}$ & $0,441 *$ \\
\hline & ID & $0,543^{* *}$ & 0,728 ** & $0,417^{*}$ & $0,372^{*}$ & - & $0,55^{* *}$ & $0,643^{* *}$ & $0,566^{* *}$ & 0,441 * & $0,669 * *$ & 0,449 ** \\
\hline & TR & $0,735^{* *}$ & $0,680 * *$ & $0,497 * *$ & $0,631 * *$ & $0,384^{*}$ & $0,711 * *$ & $0,423^{*}$ & $0,563^{* *}$ & $0,687 * *$ & $0,480 * *$ & \\
\hline & ED & - & $0,386^{*}$ & - & - & $0,359 *$ & - & $0,447^{*}$ & - & - & $0,387^{*}$ & $0,568^{* *}$ \\
\hline & T48 & $0,708^{* *}$ & $0,774 * *$ & & & & & & & & & \\
\hline & T56 & $0,761 * *$ & $0,819 * *$ & & & & & & & & & \\
\hline
\end{tabular}

Sólo se informan las correlaciones significativas. *Correlación significativa en 0,05 (dos colas). **Correlación significativa en 0,01 (dos colas). CFS: Componente de salud físico, CSM: Componente de salud mental, FF: Función física, RF: Rol físico, DC: Dolor corporal, SG: Salud general, FS: Función social, VT: Vitalidad; RE: Rol emocional, SM: Salud mental, ET: Estado de transición, EF: Estado físico, SU: Sueño, AN: Ánimo, COG: Cognición, OC: Ocio, VS: Vida social, ES: Espiritualidad, FI: Finanzas, VD: Vida doméstica, AU: Autoestima, IN: Independencia, ID: Identidad, TR: Trabajo, ED: Educación, T48: Total con 48 ítems, T56: Total con 56 ítems.

Esto significa que la variación observada en el QoL. BD-CL explica 67\% de la variación del CSM y sólo $33 \%$ restante se debe a variables no consideradas, lo que indica que ambos cuestionarios evalúan conceptos similares.

\section{Fiabilidad}

Se estimó mediante el coeficiente alpha de Cronbach. En la Tabla 3 se observa que gran parte de las dimensiones tienen, según Prieto y Delga$\mathrm{do}^{16}$, una fiabilidad adecuada ( 0,70 o superior). El error de medida especificado es importante considerarlo al momento de interpretar los puntajes individuales en cada dimensión; de este modo, el puntaje real de cada sujeto será el puntaje obtenido \pm el error de medida. Observando el alpha del total se puede establecer que el QoL. BD-CL (en sus versiones de 48 y 56 ítems) es un instrumento que presenta alta fiabilidad en la población de pacientes bipolares estudiada.
Tabla 3. Fiabilidad del cuestionario y dimensiones del QoL. BD-CL

\begin{tabular}{|lcc|}
\hline Dimensiones QoL. BD-CL & $\boldsymbol{\alpha}$ & Error de medida \\
\hline Estado físico & 0,64 & 0,40 \\
Sueño & 0,79 & 0,40 \\
Ánimo & 0,76 & 0,33 \\
Cognición & 0,69 & 0,35 \\
\hline Ocio & 0,90 & 0,25 \\
Vida Social & 0,84 & 0,32 \\
\hline Espiritualidad & 0,90 & 0,27 \\
Finanzas & 0,93 & 0,28 \\
Vida domestica & 0,95 & 0,21 \\
\hline Autoestima & 0,73 & 0,38 \\
\hline Independencia & 0,67 & 0,40 \\
\hline Identidad & 0,85 & 0,33 \\
\hline Total (48 ítems) & 0,95 & 7,43 \\
\hline Total (56 ítems) & 0,96 & 8,35 \\
\hline
\end{tabular}




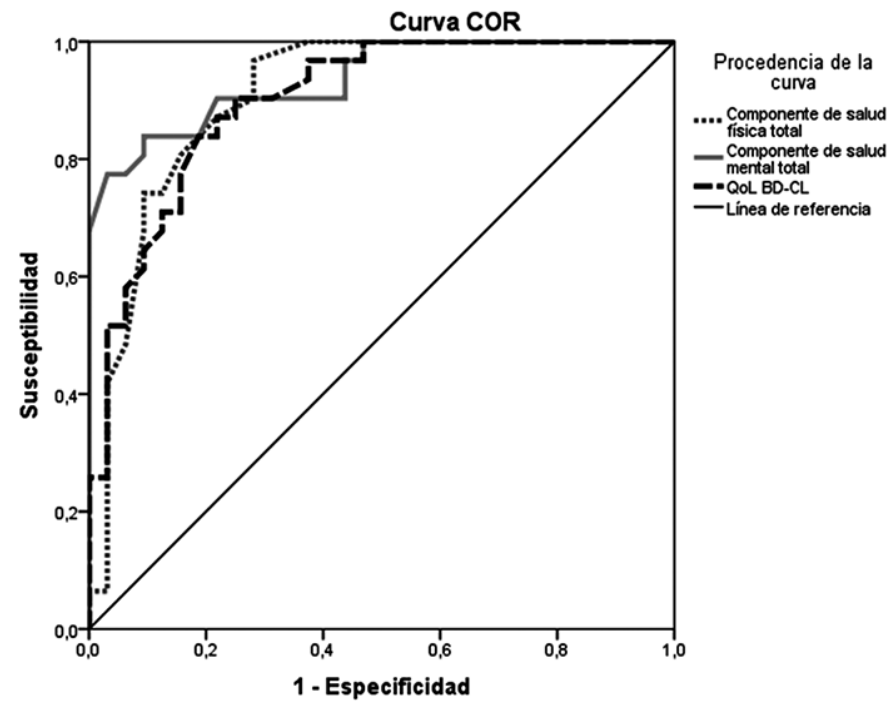

\begin{tabular}{|l|c|c|c|c|}
\hline $\begin{array}{l}\text { Variables resultado } \\
\text { de contraste }\end{array}$ & $\begin{array}{c}\text { Área bajo la } \\
\text { curva }\end{array}$ & \multirow{2}{*}{ Error típico } & \multicolumn{2}{|c|}{ Intervalo de confianza al 95\% } \\
\cline { 3 - 5 } & & 0,026 & 0,898 & 0,998 \\
\hline Componente de salud física total & $0,948^{* *}$ & 0,025 & 0,898 & 0,998 \\
\hline Componente de salud mental total & $0,948^{* *}$ & 0,027 & 0,885 & 0,991 \\
\hline QoL. BD total & $0,938^{* *}$ & \multicolumn{3}{|l}{} \\
\hline${ }^{* *} \mathrm{p}<0,01$ & \multicolumn{3}{|l}{} \\
\hline
\end{tabular}

Figura 2. Curva ROC para determinar la Utilidad diagnóstica del QoL. BD-CL.

\section{Utilidad diagnóstica}

Mediante el uso de curvas ROC se determinó que tanto el QoL. BD-CL como los dos componentes de la SF-36v.2 presentaron curvaturas pronunciadas (Figura 2). Considerando la significación estadística y los intervalos de confianza se puede inferir que las áreas estimadas en los tres casos (QoL BD-CL, CSM y CSF) son distintas de 0,5 , es decir, todos discriminan entre una mala y una buena calidad de vida. Finalmente, el punto de corte para establecer la discriminación en el QoL BD-CL es de $170 \pm 7,43$ puntos con una sensibilidad de $87,9 \%$ y especificidad de $80 \%$.

\section{Discusión}

Los resultados obtenidos permiten afirmar que el cuestionario QoL. BD-CL presenta adecuadas evidencias de fiabilidad y validez referida a criterio externo, y que permite discriminar entre una buena y mala percepción de calidad de vida, por lo que se recomienda para evaluar la calidad de vida en pacientes bipolares chilenos.

En relación al estudio original del cuestionario QoL. BD, se observó una coincidencia en las dimensiones con más alta fiabilidad (Vida doméstica, Espiritualidad y Ocio), a excepción de la dimensión Cognición, la que resultó menor en este estudio. En lo concerniente a la validez referida a criterio externo, se obtuvieron asociaciones significativas y similares a las reportadas en el estudio original entre las dimensiones del QoL. BD y las del SF- $36^{13}$.

Cabe mencionar además que se obtuvieron correlaciones significativas entre dimensiones que los creadores del instrumento no informaron y que están fundamentadas teóricamente, como -por ejemplo- la dimensión Ánimo y Autoestima con Salud mental. Sin embargo, también se registraron algunas asociaciones entre conceptos que no se miden directamente en el SF-36v.2, no descar- 
tándose que pudieran ser asociaciones espurias a pesar de su significación estadística (ejemplo: Vida doméstica y Función social); futuros estudios podrán responder satisfactoriamente a esta hipótesis.

Por otra parte, se estableció que los pacientes bipolares obtienen puntajes significativamente menores que el grupo no clínico, tanto en el cuestionario SF-36v.2 como en el QoL.BD-CL $(\mathrm{p}<0,001)$. Esta diferencia en la percepción de la calidad de vida es congruente con los resultados de estudios previos ${ }^{4,12,17,18,19}$. Es importante destacar que se obtuvo información referida a la sensibilidad y especificidad del instrumento, aspecto que no se había informado previamente. El resultado de ello fue establecer un punto de corte de $170 \pm$ 7,43 puntos, límite que permitir distinguir entre una buena y mala percepción de calidad de vida, lográndose clasificar correctamente a 82,5\% de los casos estudiados. Esto no sólo es estadísticamente significativo sino también teóricamente relevante, ya que el puntaje de corte (incluso considerando el error de medida) logró clasificar mayoritariamente a los pacientes bipolares con una mala calidad de vida y a los sujetos no clínicos con una buena calidad de vida.

Si bien, el QoL.BD-CL y la SF-36v.2 presentan similar utilidad diagnóstica, el aporte que realiza el primero tiene relación con aspectos clínicos, dado que es capaz de entregar información específica del trastorno bipolar explorando aquellas áreas más afectadas, convirtiendo al Qol.BD-CL en un instrumento riguroso desde lo estadístico y útil desde lo clínico. De esta manera, puede ayudar a los profesionales de la salud mental a focalizar las diversas intervenciones y contribuir en investigaciones donde se evalúen diferentes tratamientos, ya sean farmacológicos o psicosociales. Además, como parte de las ventajas que presenta la utilización del QoL.BD-CL se puede mencionar que tiene instrucciones e ítems simples de comprender; no presenta mayor complicación al momento de aplicar ni de puntuar; es fácil de responder y toma poco tiempo administrarlo.

Por último, es necesario señalar que la principal limitación del presente estudio tiene relación con las características de la muestra, tanto por el tamaño como por su localización, ya que sólo se trabajó con pacientes de un sector de Santiago. El mejorar estas condiciones permitiría realizar otros análisis que siguieran aportando evidencias de validez y fiabilidad del QoL. BD-CL. Además, se podría realizar una estandarización del instrumento para obtener normas adecuadas a la población chilena y realizar estudios donde se evalúe la calidad de vida con el QoL BD-CL entre las distintas fases del trastorno bipolar y entre distintas patologías psiquiátricas para establecer la especificidad del instrumento.

\section{Anexo 1. Cuestionario de Calidad de Vida en el Trastorno Bipolar (QoL BD-CL)}

Morgado C, Tapia T, Ivanovic-zuvic F, Antivilo A.

Los siguientes ítems corresponden a una serie de experiencias, comportamientos y sentimientos relacionados con la calidad de vida. Por favor, cuéntenos acerca de su calidad de vida calificando las afirmaciones que se presentan a continuación, indicando su grado de acuerdo o desacuerdo con cada una de ellas. No se tome mucho tiempo en cada ítem, ya que son sus primeras impresiones las que nos interesa.

Marque con un círculo el número que mejor describa su experiencia durante los ÚLTIMOS 7 DÍAS, tal como se señala a continuación.

\begin{tabular}{|c|c|c|c|c|c|c|c|}
\hline \multicolumn{2}{|c|}{ Muy en Desacuerdo } & En Desacuerdo & \multicolumn{2}{|c|}{$\begin{array}{c}\text { Ni de acuerdo ni en } \\
\text { desacuerdo }\end{array}$} & De acuerdo & \multicolumn{2}{|c|}{ Muy de acuerdo } \\
\hline & 1 & & \multicolumn{2}{|c|}{3} & 4 & \multicolumn{2}{|c|}{5} \\
\hline \multicolumn{3}{|c|}{ Durante los últimos 7 días, yo... } & $\begin{array}{c}\text { Muy en } \\
\text { Desacuerdo }\end{array}$ & \begin{tabular}{|c} 
En \\
Desacuerdo
\end{tabular} & $\begin{array}{l}\text { Ni de acuerdo ni } \\
\text { en desacuerdo }\end{array}$ & $\begin{array}{c}\text { De } \\
\text { acuerdo }\end{array}$ & $\begin{array}{l}\text { Muy de } \\
\text { acuerdo }\end{array}$ \\
\hline 1 & \multicolumn{2}{|c|}{ He tenido mucha energía } & 1 & 2 & 3 & 4 & 5 \\
\hline 2 & \multicolumn{2}{|c|}{ Siento que he hecho suficiente ejercicio } & 1 & 2 & 3 & 4 & 5 \\
\hline 3 & \multicolumn{2}{|c|}{ Me he sentido bien físicamente } & 1 & 2 & 3 & 4 & 5 \\
\hline 4 & \multicolumn{2}{|c|}{ He estado satisfecho con mi vida sexual } & 1 & 2 & 3 & 4 & 5 \\
\hline 5 & \multicolumn{2}{|c|}{ Me he despertado con energía } & 1 & 2 & 3 & 4 & 5 \\
\hline
\end{tabular}




\begin{tabular}{|c|c|c|c|c|c|c|}
\hline \multicolumn{2}{|c|}{ Durante los últimos 7 días, yo... } & \multirow{2}{*}{\begin{tabular}{|c|}
$\begin{array}{c}\text { Muy en } \\
\text { Desacuerdo }\end{array}$ \\
1
\end{tabular}} & \multirow{2}{*}{$\begin{array}{c}\text { En } \\
\text { Desacuerdo } \\
2\end{array}$} & \multirow{2}{*}{\begin{tabular}{|c|}
$\begin{array}{c}\text { Ni de acuerdo } \mathrm{ni} \\
\text { en desacuerdo }\end{array}$ \\
3 \\
\end{tabular}} & \multirow{2}{*}{$\begin{array}{c}\begin{array}{c}\text { De } \\
\text { acuerdo }\end{array} \\
4\end{array}$} & \multirow{2}{*}{\begin{tabular}{|c|}
$\begin{array}{c}\text { Muy de } \\
\text { acuerdo }\end{array}$ \\
5
\end{tabular}} \\
\hline 6 & $\begin{array}{l}\text { NO he tenido problemas para levantar- } \\
\text { me por las mañanas }\end{array}$ & & & & & \\
\hline 7 & He dormido lo suficiente & 1 & 2 & 3 & 4 & 5 \\
\hline 8 & $\begin{array}{l}\text { He mantenido una rutina en mis hora- } \\
\text { rios de sueño }\end{array}$ & 1 & 2 & 3 & 4 & 5 \\
\hline 9 & Me he sentido feliz & 1 & 2 & 3 & 4 & 5 \\
\hline 10 & He disfrutado las cosas igual que siempre & 1 & 2 & 3 & 4 & 5 \\
\hline 11 & $\begin{array}{l}\text { Me he sentido capaz de enfrentar situa- } \\
\text { ciones difíciles }\end{array}$ & 1 & 2 & 3 & 4 & 5 \\
\hline 12 & Me he sentido emocionalmente estable & 1 & 2 & 3 & 4 & 5 \\
\hline 13 & He pensado con claridad & 1 & 2 & 3 & 4 & 5 \\
\hline 14 & He tenido buena concentración & 1 & 2 & 3 & 4 & 5 \\
\hline 15 & NO he tenido dificultades con mi memoria & 1 & 2 & 3 & 4 & 5 \\
\hline 16 & He hecho planes sin dificultad. & 1 & 2 & 3 & 4 & 5 \\
\hline 17 & $\begin{array}{l}\text { He disfrutado mis actividades de re- } \\
\text { creación }\end{array}$ & 1 & 2 & 3 & 4 & 5 \\
\hline 18 & $\begin{array}{l}\text { He estado interesado en mis actividades } \\
\text { de recreación }\end{array}$ & 1 & 2 & 3 & 4 & 5 \\
\hline 19 & $\begin{array}{l}\text { Me he entretenido en mis actividades } \\
\text { de recreación }\end{array}$ & 1 & 2 & 3 & 4 & 5 \\
\hline 20 & He expresado mi creatividad & 1 & 2 & 3 & 4 & 5 \\
\hline 21 & $\begin{array}{l}\text { He disfrutado pasar tiempo con otras } \\
\text { personas }\end{array}$ & 1 & 2 & 3 & 4 & 5 \\
\hline 22 & $\begin{array}{l}\text { He estado interesado en mis relaciones } \\
\text { sociales }\end{array}$ & 1 & 2 & 3 & 4 & 5 \\
\hline 23 & He tenido amistades significativas & 1 & 2 & 3 & 4 & 5 \\
\hline 24 & $\begin{array}{l}\text { He sido capaz de compartir mis senti- } \\
\text { mientos o problemas con un/a amigo/a }\end{array}$ & 1 & 2 & 3 & 4 & 5 \\
\hline 25 & $\begin{array}{l}\text { Me he sentido satisfecho con mi aspecto } \\
\text { espiritual }\end{array}$ & 1 & 2 & 3 & 4 & 5 \\
\hline 26 & $\begin{array}{l}\text { He expresado mi espiritualidad tal como } \\
\text { deseo }\end{array}$ & 1 & 2 & 3 & 4 & 5 \\
\hline 27 & $\begin{array}{l}\text { He practicado mi espiritualidad tal } \\
\text { como deseo }\end{array}$ & 1 & 2 & 3 & 4 & 5 \\
\hline 28 & $\begin{array}{l}\text { He mantenido una rutina con respecto a } \\
\text { mi espiritualidad }\end{array}$ & 1 & 2 & 3 & 4 & 5 \\
\hline 29 & $\begin{array}{l}\text { He tenido dinero suficiente para mis } \\
\text { necesidades básicas }\end{array}$ & 1 & 2 & 3 & 4 & 5 \\
\hline 30 & $\begin{array}{l}\text { He tenido dinero suficiente para cosas } \\
\text { extras }\end{array}$ & 1 & 2 & 3 & 4 & 5 \\
\hline 31 & $\begin{array}{l}\text { Me he sentido seguro respecto de mi } \\
\text { situación financiera }\end{array}$ & 1 & 2 & 3 & 4 & 5 \\
\hline 32 & No he tenido problemas con mis deudas & 1 & 2 & 3 & 4 & 5 \\
\hline 33 & He realizado las labores diarias del hogar & 1 & 2 & 3 & 4 & 5 \\
\hline 34 & He sido organizado en mi hogar & 1 & 2 & 3 & 4 & 5 \\
\hline 35 & He mantenido mi casa ordenada & 1 & 2 & 3 & 4 & 5 \\
\hline 36 & He mantenido mi casa limpia & 1 & 2 & 3 & 4 & 5 \\
\hline 37 & Me he sentido respetado & 1 & 2 & 3 & 4 & 5 \\
\hline 38 & Me he sentido aceptado por otros & 1 & 2 & 3 & 4 & 5 \\
\hline
\end{tabular}


Propiedades psicométricas del "Quality of Life Bipolar Disorder" en español - C. Morgado et al

\begin{tabular}{|l|l|c|c|c|c|c|}
\hline \multicolumn{2}{|l|}{ Durante los últimos 7 días, yo... } & $\begin{array}{c}\text { Muy en } \\
\text { Desacuerdo }\end{array}$ & $\begin{array}{c}\text { En } \\
\text { Desacuerdo }\end{array}$ & $\begin{array}{c}\text { Ni de acuerdo ni } \\
\text { en desacuerdo }\end{array}$ & $\begin{array}{c}\text { De } \\
\text { acuerdo }\end{array}$ & $\begin{array}{c}\text { Muy de } \\
\text { acuerdo }\end{array}$ \\
\hline 39 & $\begin{array}{l}\text { Me he sentido tan valioso como los } \\
\text { demás }\end{array}$ & 1 & 2 & 4 & 5 \\
\hline 40 & $\begin{array}{l}\text { Me he sentido capaz de enfrentar mi } \\
\text { diagnóstico }\end{array}$ & 1 & 2 & 3 & 5 \\
\hline 41 & Me he sentido libre & 1 & 2 & 3 & 4 & 5 \\
\hline 42 & Me he sentido seguro en mi hogar & 1 & 2 & 3 & 5 \\
\hline 43 & $\begin{array}{l}\text { Me he transportado de un lugar a otro } \\
\text { libremente (ej. Conduciendo, usando } \\
\text { transantiago) }\end{array}$ & 1 & 2 & 3 & 5 \\
\hline 44 & $\begin{array}{l}\text { He sentido que los demás me permiten } \\
\text { ser independiente }\end{array}$ & 1 & 2 & 3 & 4 & 5 \\
\hline 45 & Estoy seguro de mí mismo & 1 & 2 & 3 & 4 & 5 \\
\hline 46 & Me reconozco a mí mismo & 1 & 2 & 3 & 4 & 5 \\
\hline 47 & $\begin{array}{l}\text { He tenido una idea clara de lo que quie- } \\
\text { ro y lo que No quiero }\end{array}$ & 1 & 2 & 3 & 4 & 5 \\
\hline 48 & He tenido control sobre mi vida & 1 & 2 & 3 & 4 & 5 \\
\hline
\end{tabular}

¿Está usted actualmente trabajando de manera remunerada y/o voluntaria?

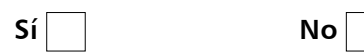

Si su respuesta es Sí responda las siguientes 4 preguntas

\begin{tabular}{|l|l|c|c|c|c|c|}
\hline \multicolumn{2}{|l|}{ Durante los últimos 7 días, yo... } & $\begin{array}{c}\text { Muy en } \\
\text { Desacuerdo }\end{array}$ & $\begin{array}{c}\text { En } \\
\text { Desacuerdo }\end{array}$ & $\begin{array}{c}\text { Ni de acuerdo ni } \\
\text { en desacuerdo }\end{array}$ & $\begin{array}{c}\text { De } \\
\text { acuerdo }\end{array}$ & $\begin{array}{c}\text { Muy de } \\
\text { acuerdo }\end{array}$ \\
\hline 49 & $\begin{array}{l}\text { Me he sentido seguro con mis habilida- } \\
\text { des en el trabajo }\end{array}$ & 1 & 2 & 3 & 4 & 5 \\
\hline 50 & $\begin{array}{l}\text { He cumplido con los requerimientos } \\
\text { del trabajo }\end{array}$ & 1 & 2 & 3 & 4 & 5 \\
\hline 51 & $\begin{array}{l}\text { He estado satisfecho con la calidad de } \\
\text { mi trabajo }\end{array}$ & 1 & 2 & 3 & 4 & 5 \\
\hline 52 & He sido confiable en el trabajo & 1 & 2 & 3 & 4 & 5 \\
\hline
\end{tabular}

¿Está usted actualmente estudiando?

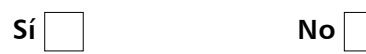

Si su respuesta es Sí responda las siguientes 4 preguntas

\begin{tabular}{|l|l|c|c|c|c|c|}
\hline \multicolumn{2}{|l|}{ Durante los últimos 7 días, yo... } & $\begin{array}{c}\text { Muy en } \\
\text { Desacuerdo }\end{array}$ & $\begin{array}{c}\text { En } \\
\text { Desacuerdo }\end{array}$ & $\begin{array}{c}\text { Ni de acuerdo ni } \\
\text { en desacuerdo }\end{array}$ & $\begin{array}{c}\text { De } \\
\text { acuerdo }\end{array}$ & $\begin{array}{c}\text { Muy de } \\
\text { acuerdo }\end{array}$ \\
\hline 53 & He disfrutado mis estudios & 1 & 2 & 3 & 4 & 5 \\
\hline 54 & $\begin{array}{l}\text { Me he sentido seguro de terminar mis } \\
\text { estudios }\end{array}$ & 1 & 2 & 3 & 4 & 5 \\
\hline 55 & He mantenido mi rendimiento habitual & 1 & 2 & 3 & 4 & 5 \\
\hline 56 & $\begin{array}{l}\text { He organizado mis actividades educati- } \\
\text { vas adecuadamente }\end{array}$ & 1 & 2 & 3 & 4 & 5 \\
\hline
\end{tabular}




\section{Referencias}

1. Vieta E. Trastornos bipolares y esquizoafectivos. En: Vallejo J. Director, Introducción a la psicopatología y la psiquiatría. Barcelona, España: Editorial Elsevier Masson; 2011. p. 258-78.

2. López A, Murray C. The global burden of disease, 19902020. Nature Medicine 1998; 4 (11): 1241-3.

3. Vieta E, Blasco-Colmenares E, Figueira M, Lanogosch J, Moreno-Manzanaros M, Medina E. Clinical management and burden of bipolar diosorder: a multinational longitudinal study (WAVE-bdStudy). BMC Psychiatry 2011; 11 (58). Disponible en http://www.biomedcentral. com/1471-244X/11/58 [Consultado el 30 de septiembre de 2012].

4. Gutiérrez L. Curso evolutivo y calidad de vida en el trastorno bipolar. Tesis doctoral; 2008. Facultad de Medicina. Universidad de Granada.

5. Retamal P. Manejo psicoterapéutico de la enfermedad bipolar. En Cómo enfrentar la enfermedad bipolar, guía para el paciente y la familia $\left(2^{\circ}\right.$ Edición.) Santiago, Buenos Aires: Mediterráneo; 2010. p. 82-91.

6. Ministerio de Salud Acceso Universal Garantías Explícitas. Más enfermedades AUGE Chile avanza con todos 2013. Disponible en http://www.minsal.cl/portal/url/ page/minsalcl/g_gesauge/auge80.html [Consultado el 17 de julio de 2013].

7. The WHOQOL Group. The World Health Organization Quality of Life assessment (WHOQOL): position paper from the World Health Organization. Soc Sci Med 1995; 41: 1403-9.

8. Schwartzmann L. Calidad de vida relacionada con la salud: aspectos conceptuales. Ciencia y Enfermería 2003; IX (2): 9-21.

9. Casas J, Repullo J, Pereira J. Medidas de calidad de vida relacionada con la salud. Conceptos básicos, construcción y adaptación cultural. Medicina Clínica 2001; 116 (29): 789-96.

10. Alvarado R, Muñoz K. Estudio psicométrico del Cuestionario Sevilla para valorar la calidad de vida en personas con trastornos mentales severos, en una muestra chilena. Rev Chil Neuro-Psiquiat 2006; 44 (4): 249-57.

11. Soto A. Incorporación de estudios de calidad de vida relacionada con la salud en los ensayos clínicos: bases y recomendaciones prácticas. Uso de una lista-guía para su correcto diseño y/o evaluación. Anales de Medicina Interna (Madrid) 2003; 20 (12): 633-44.

12. Michalak E, Yatham L, Lam R. Quality of life in bipolar disorder: A review of the literature. Health and Quality of Life Outcomes 2005; 3 (72). Disponible en http:// www.hqlo.com/content/3/1/72 [Consultado el 29 de septiembre de 2012].

13. Michalak E, Murray G \& the Collaborative Research Team to Study Psychosocial Issues in Bipolar Disorder. Development of the QoL.BD: a disorder specific scale to assess quality of life in bipolar disorder. Bipolar Disorders 2010; 12: 727-40. Disponible en http://www.crestbd. $\mathrm{ca} /$ research-projects/quality-of-life-scale/ [Consultado el 23 de agosto de 2012].

14. "Crestbd" (s.f.). QoL. BD-The FaQs. Disponible en http://www.crestbd.ca/research-projects/quality-of-lifescale/qol-bd-scale-faqs/ [Consultado el 23 de agosto de 2013].

15. Olivares-Tirado P. Estado de salud de beneficiarios del sistema de salud de Chile: 2004-2005. 2006: 1-40. Disponible en http://www.supersalud.gob.cl/documentacion/569/articles-1062_recurso_1.pdf [Consultado el 20 de octubre de 2012].

16. Prieto G, Delgado A. Fiabilidad y Validez. Papeles del Psicólogo 2010; 1 (31): 67-74.

17. Sierra P, Livianos L, Rojo L. Quality of life for patients with bipolar disorder: relationship with clinical and demographic variables. Bipolar Disorder 2005; 7: 15965.

18. Michalak E, Yatham L, Kolesar S, Lam R. Bipolar disorder and quality of life: A patient-centered perspective. Quality of Life Research 2006; 15: 25-37.

19. Ishak W, Brown K, Aye S, Kahloon M, Mobaraki S, Hanna R. Health-related quality of life in bipolar disorder. Bipolares Disorder 2012; 14: 6-18. 